\title{
Uso e manejo do solo e seus impactos sobre a qualidade física
}

\author{
Diane C. Stefanoski ${ }^{1}$, Glenio G. Santos ${ }^{1}$, Robélio L. Marchão², \\ Fabiano A. Petter ${ }^{3}$ \& Leandro P. Pacheco ${ }^{4}$
}

\begin{abstract}
RESUMO
O uso racional do solo tem sido objeto de estudo e discussões em função da busca de alternativas tecnológicas que possibilitem o manejo correto do solo e, consequentemente, uma agricultura sustentável. Objetivou-se, com este trabalho, levantar dados sobre a qualidade físico-hídrica e sua relação com os impactos gerados pelo manejo do solo por meio de uma revisão bibliográfica, contribuindo para melhor conhecimento das informações disponíveis na literatura. $\mathrm{O}$ uso de indicadores de qualidade física do solo permite designar práticas adequadas de manejo do solo. Uma análise crítica dos indicadores de qualidade física do solo revela que o índice proposto por Reynolds et al. (2002) possui limitações quanto ao uso, o intervalo hídrico ótimo apresenta bons resultados e o índice $\mathrm{S}$ necessita de mais trabalhos com o uso de $\mathrm{h}$ (tensão de água no solo) como variável independente. Assim, os indicadores de qualidade física do solo são úteis para avaliação do estado de conservação do solo possibilitando a gestão segura dos recursos naturais; todavia, os indicadores existentes apresentam, como inconvenientes, a elevada complexidade de execução e os custos de obtenção, o que configura oportunidade para o desenvolvimento de novos indicadores de qualidade física do solo.
\end{abstract}

Palavras-chave: sistemas de manejo, compactação, estrutura do solo, indicadores de qualidade física

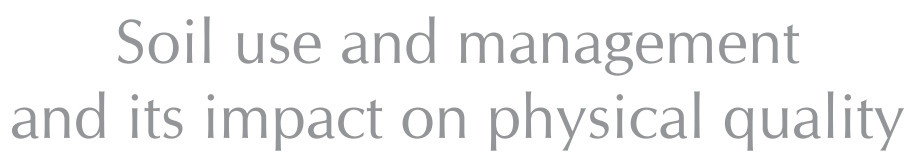

\begin{abstract}
The rational use of the soil has been the subject of study and discussion due to search for technological alternatives that allow the adequate management of the soil and consequently, a sustainable agriculture. The objective of this study was to collect data about the hydro-physical quality and its relation to the impacts caused by soil management through a literature review, contributing to better understanding of existing information in the literature. The use of indicators of physical quality of soil permit the designation of most appropriate soil management practices, especially those exposed to intensive agriculture. A critical analysis of indicators of soil quality show that the index proposed by Reynolds et al. (2002) has limitations about its use, the optimal hydric interval presents good results, and the $\mathrm{S}$ index needs more works with the use of $\mathrm{h}$ (soil-water suction) as the independent variable. Thus, the indicators of physical quality of soil are useful for evaluating the state of soil conservation, to making possible the secure management of natural resources, however, the existing indicators present very high complexity of the implementation and acquisition costs, which configures opportunity for the development of new indicators of physical quality of soil.
\end{abstract}

Key words: management systems, compaction, soil structure, indicators of physical quality

PPGSN/UFPI, Campus Professora Cinobelina Elvas, BR 135, km três, CEP 64900-000, Bom Jesus, PI. Fone: (89) 3562-2468. E-mail: diane_stefanoski@hotmail.com; gleniogm@gmail.com

${ }^{2}$ Embrapa Cerrados, C.P. 8223, CEP 73310-970, Planaltina, DF. Fone: (61) 3388-9898. E-mail: robelio.leandro@cpac.embrapa.br

${ }^{3}$ UFMT, Campus Universitário de Sinop, Av. Alexandre Ferronato, 1200, Reserva 35, Setor Industrial, CEP 78557-267, Sinop, MT. Fone: (66) 8103-4611. E-mail: petter@ufpi.edu.br

${ }^{4}$ UFMT, Campus Universitário de Rondonópolis, Parque Sagrada Família, CEP 78735901, Rondonópolis, MT. Fone: (66) 3410-4063. E-mail: leandroppacheco@terra.com.br 


\section{INTRODUÇÃO}

Ao se discutir a sustentabilidade da produção agropecuária chamam a atenção o uso do solo com a agricultura tradicional, com preparo contínuo do solo, e a extensão de áreas de pastagens degradadas (Macedo, 2009). Dos componentes do manejo o preparo do solo talvez seja a atividade que mais exerce influência nos atributos indicadores da qualidade física do solo, pois atua diretamente na sua estrutura (Hamza \& Anderson, 2005).

$\mathrm{O}$ conceito de qualidade física do solo (QFS) engloba o conhecimento de propriedades e processos relativos à habilidade do solo em manter efetivamente os serviços ambientais ou serviços ecossistêmicos essenciais à saúde do ecossistema (MEA, 2005), cujo estudo é realizado através de indicadores físicos da qualidade do solo responsáveis pela avaliação da sua estrutura.

Segundo Singer \& Ewing (2000), os atributos mais utilizados como indicadores de QFS deveriam ser aqueles que consideram a profundidade efetiva de enraizamento, porosidade total, distribuição e tamanho dos poros, distribuição do tamanho das partículas, densidade do solo, resistência do solo à penetração das raízes, intervalo hídrico ótimo, índice de compressão e estabilidade dos agregados.

Contudo, há outros indicadores de QFS, como a curva de retenção de água do solo, a condutividade hidráulica, a porosidade, o ponto de inflexão e as características de retenção de água do solo; que tornam o processo de avaliação da qualidade física mais simples, rápido e menos complexo (Santos et al., 2011a).

Ao considerar que o recurso solo é limitado e que alguns de seus componentes requerem períodos de tempo prolongados para serem restaurados, a previsão do grau das perturbações ambientais provocadas pelo manejo inadequado das atividades agropecuárias se torna essencial. Técnicas que visem avaliar a qualidade de um solo de forma simples e confiável ainda são objeto de investigações, e resultados sistemáticos deste monitoramento representam papel central em estudos sobre qualidade do solo (QS).

Nesse sentido, ainda existe um campo a ser explorado no que concerne ao desenvolvimento de novos indicadores para a avaliação da QFS, principalmente no caso dos solos que compõem os diferentes biomas brasileiros, ainda pouco explorados, notadamente no tocante aos estudos associados ao uso de atributos físico-hídricos como forma de aferição de sua sustentabilidade produtiva (Santos, 2010).

Diante do exposto, esta revisão objetivou explorar os dados disponíveis sobre o tema, contribuindo para uma análise mais abrangente acerca dos trabalhos existentes na literatura referentes à qualidade física do solo e sua relação com os impactos gerados pelo seu manejo.

\section{Estrutura DO SOLO}

A qualidade estrutural do solo refere-se ao arranjo das partículas do solo constituindo um ambiente dinâmico, cuja alteração determinará um novo comportamento dos processos que ocorrem no solo (Ferreira, 2010).

A estrutura é boa indicadora da QS devido a sua sensibilidade às práticas de manejo adotadas. De modo geral, o solo mantido em estado natural, sob vegetação nativa, apresenta características físicas como estrutura, permeabilidade, densidade e porosidade do solo adequadas ao desenvolvimento normal das plantas (Andreola et al., 2000). Na medida em que se intensifica o uso agrícola os atributos físico-hídricos do solo sofrem alterações, geralmente adversas ao crescimento vegetal, que ficam mais nítidas quando os sistemas de uso são comparados com o estado do solo ainda sob vegetação natural (Santos, 2010, Santos et al., 2011a).

Segundo Aguiar (2008), a estrutura pode ser avaliada pela densidade do solo, macro e microporosidade, estabilidade de agregados, resistência à penetração e infiltração da água no solo. Já para Reinert et al. (2006), a estrutura é avaliada pelos atributos que lhe dão forma, isto é, densidade do solo; geometria, tamanho e continuidade de poros; infiltração; retenção de água e aeração.

De acordo com Kiehl (1979), a densidade do solo deve permanecer no intervalo entre 1,1 a $1,6 \mathrm{Mg} \mathrm{m}^{-3} \mathrm{em}$ solos minerais, assumindo valores superiores a $1,6 \mathrm{Mg} \mathrm{m}^{-3} \mathrm{em}$ solos de textura arenosa. Camargo \& Alleoni (1997) assinalaram como crítico, em solos variando de franco-argilosos a argilosos, o valor de densidade na ordem de $1,55 \mathrm{Mg} \mathrm{m}^{-3}$. Já para o cultivo da soja em solos franco-argilo-arenosos, Beutler et al. (2005) afirmaram que a densidade ótima deve ser da ordem de $1,23 \mathrm{Mg} \mathrm{m}^{-3}$.

De maneira similar, a porosidade de aeração ou porosidade livre de água (Libardi, 2000) é diretamente afetada pelos valores de densidade e pelo conteúdo de água no solo. Tomando a porosidade total como um valor fixo, pode-se dizer que uma parte pode ser ocupada por água e a outra por ar. Quando a porosidade de aeração é menor que $10 \%$, a taxa de fluxo de oxigênio em direção ao sistema radicular das plantas é severamente prejudicada afetando, assim, os processos fisiológicos/metabólicos e, consequentemente, o crescimento das raízes das plantas (Leão, 2002).

Lanzanova et al. (2007) constataram, ao estudar atributos físicos do solo em sistema de integração lavoura-pecuária sob plantio direto, que a microporosidade do solo não foi significativamente alterada pelos sistemas de manejo das pastagens em nenhuma das camadas de solo estudadas; contudo, a macroporosidade e a porosidade total sofreram influência do pisoteio bovino na camada superficial, ambas diferindo da área não pastejada. Já Cassol (2003) observou aumento na infiltração de água diretamente proporcional ao incremento da altura de resíduo da pastagem, evidenciando a degradação da QS quando se utiliza elevada pressão de pastejo.

Outro atributo do solo que merece ser destacado é a estabilidade de agregados. De acordo com estudos sobre a influência do manejo na agregação do solo, Tisdall \& Oades (1980) indicaram que a influência do manejo do solo na estabilidade dos microagregados menores que $0,25 \mathrm{~mm}$ não é tão importante; entretanto, deve ser considerado que as partículas de argilas dispersas no solo são liberadas lentamente e agem como uma nuvem expansiva envolvendo os agregados (Arnold, 1978). Assim, a argila dispersa pode bloquear os poros que redistribuem ou armazenam água no solo, resultando em estruturas indesejáveis funcionalmente semelhantes a superfícies compactadas (Tisdall \& Oades, 1982). 
Nesse contexto estudos têm demonstrado que a resistência dos agregados e a friabilidade do solo são influenciados por diversos fatores tais como: umidade do solo, tipo e teor de argila, mineralogia, metais polivalentes, carbonato de cálcio, óxidos e hidróxidos de ferro, alumínio e manganês, composição e concentração da solução do solo, ciclos de umedecimento e secagem do solo, exsudatos orgânicos de plantas, substâncias orgânicas provenientes da ação de micro-organismos e outros compostos orgânicos (Bastos et al., 2005).

Em geral, o aumento no teor de argila induz o incremento na resistência tênsil dos agregados e, portanto, redução da friabilidade (Kemper et al., 1987), o que também se observa ao reduzir proporcionalmente os teores de carbono orgânico no solo (Tormena et al., 2008). A elevação da resistência tênsil em razão do aumento no teor de argila parece ser função do incremento do número de cargas elétricas, o que favorece a formação de ligações entre partículas minerais e/ou partículas minerais e orgânicas (Imhoff, 2002).

Dufranc et al. (2004) constataram como principais agentes ligantes em dois Latossolos Vermelhos em sistema de plantio direto, a comunidade bacteriana, os teores de ferro e de potássio e a matéria orgânica. Por outro lado, alguns autores atestam que os óxidos de ferro exercem pouca ou nenhuma influência na agregação do solo (Muggler et al., 1997), ou que o efeito desses agentes agregantes é devido à sua interação com a matéria orgânica do solo e/ou teor de argila (Guérif, 1990).

Em estudo para se avaliar a agregação e estoques de carbono sob diferentes práticas de manejo agrícola, Vezzani \& Mielniczuk (2011) verificaram que houve recuperação da agregação do solo e estoques de carbono próximo das condições naturais de Campo Nativo com o uso e manejo de sistemas de culturas por 15 a 17 anos sem revolvimento do solo associado ao incremento de carbono.

Costa et al. (2009) constataram que o revolvimento do solo com arado e grade ou escarificador reduz o teor de carbono de compostos orgânicos, a estabilidade de agregados, a densidade e a capacidade de campo, porém, aumenta a porosidade total, a macroporosidade e a capacidade de aeração em comparação com o sistema de plantio direto. Dantas et al. (2012) por sua vez, concluíram que o cultivo anual resulta em maior degradação física do solo, se comparado ao cultivo perene, em função da redução na condutividade hidráulica no solo e na estabilidade de agregados maiores que $2,0 \mathrm{~mm}$, com elevação nos valores de densidade do solo.

De acordo com Campos et al. (1995), o contínuo aporte de material orgânico é fonte de energia para a atividade microbiana, que atua como agente de estabilização dos agregados. Além disso, a matéria orgânica, em razão de sua estrutura complexa e longas cadeias de carbono, agrega partículas minerais (Dufranc et al., 2004), com efeito direto na formação e na estabilização dos agregados (Braida et al., 2010), sendo uma importante propriedade para se estimar a qualidade física dos solos (Ribon \& Tavares Filho, 2008).

Dessa forma, a adoção de sistemas de manejo que mantenham a proteção do solo pelo contínuo aporte de resíduos orgânicos é essencial para manutenção e/ou melhoria da estrutura do solo.

\section{Compactação do solo}

Na compactação do solo há aumento de massa por unidade de volume, resultando em aumento na densidade, na resistência à penetração de raízes e na microporosidade relativa, o que contribui para redução linear da porosidade total e da macroporosidade (Beutler et al., 2005).

Com relação a estudos sobre compactação do solo, Silva et al. (2005) afirmaram que a deposição de argila nas paredes dos poros e sobre os grãos de quartzo ocasiona um arranjamento mais compacto de partículas, aumentando o adensamento das camadas subsuperficiais. Este fenômeno é mais expressivo em solos cuja argila está em um maior grau de dispersão, migrando facilmente sob condições de chuva ou irrigação (Helalia et al., 1988).

A compactação do solo pelo tráfego de máquinas, originada da compressão do solo insaturado, é a principal causa da degradação física dos solos agrícolas, e aumenta com a intensidade de tráfego em condições inadequadas de umidade do solo (Flowers \& Lal, 1998). A susceptibilidade à compactação pode ser alterada pelo acúmulo de matéria orgânica, porém, a textura do solo e seus efeitos associados à retenção de água, coesão e densidade do solo determinarão a magnitude e o tipo de efeito (Braida et al., 2010).

A compactação, devida à atividade agropecuária, também pode causar modificações na retenção de água do solo, decorrentes de alterações sofridas na distribuição do diâmetro de poros, principalmente redução da macroporosidade (Figueiredo et al., 2009). Todavia, a disponibilidade de água para as plantas depende do nível de compactação e da distribuição do diâmetro de poros resultante (Hillel, 1980).

Albuquerque et al. (2001) relataram que a compactação causada pelo excessivo tráfego de máquinas e implementos agrícolas e pelo pisoteio animal em áreas sob integração lavoura-pecuária - ILP, tem sido uma das principais causas da degradação da capacidade produtiva de solos agrícolas; entretanto, Moreira et al. (2012), ao avaliar a densidade do solo, porosidade de aeração e a permeabilidade do solo ao ar, concluíram que, após oito anos de ILP, não se confirmou a hipótese de que o aumento do pisoteio animal diminui a QFS. Santos et al. (2011a) afirmam que são escassos experimentos de longa duração que permitam observar as alterações na QS resultantes dos efeitos acumulados dos sistemas de manejo.

Nesse contexto, a resistência do solo à penetração se torna um importante atributo físico indicador da QFS, pois se relaciona com outros atributos indicadores do grau de compactação (Ribon \& Tavares Filho, 2008). Embora varie com o solo e com a espécie cultivada, a resistência do solo à penetração é ocasionada por atividades antrópicas, de forma continuada e mal-planejada. Marchão et al. (2007) demonstraram que o atributo umidade apresenta relação inversamente proporcional aos valores de resistência à penetração obtidos em condições de campo.

Valores críticos de resistência à penetração podem variar de 1,5 MPa a 4,0 MPa (Rosolem et al., 1999); no entanto, valores próximos a $2 \mathrm{MPa}$ são, de maneira geral, aceitos como impeditivos ao crescimento radicular (Blainski et al., 2008). Todavia, para a cultura da soja Beutler et al. (2007) verificaram redução na produtividade de grãos em solos 
com intervalo de resistência à penetração entre 1,30 e 1,64 MPa; logo, a compactação em razão do sistema de manejo do solo e das culturas pode implicar em alterações físicas que determinam condições ideais ou limitantes ao desenvolvimento vegetal (Collares et al., 2008). Assim, as práticas de manejo e de conservação do solo e da água devem ser planejadas e executadas procurando-se manter, ou mesmo melhorar, seus atributos de modo a aumentar a capacidade do solo em sustentar uma produtividade competitiva, sob aspectos físicos, químicos e biológicos, sem comprometer a qualidade do solo e da água.

\section{Indicadores da Qualidade Física do Solo}

Doran \& Parkin (1994) classificam os atributos físicos do solo como intermediários e permanentes. Exemplos dos primeiros, alteráveis pelo tipo de manejo do solo, são: densidade do solo, resistência à penetração, permeabilidade, aeração, agregação, porosidade e umidade do solo. Textura do solo, mineralogia, densidade de partículas e cor, podem ser elencadas como atributos físicos permanentes, inerentes às propriedades de cada solo e que servem para classificá-lo pedologicamente. Em geral, os atributos físicos intermediários são os mais utilizados como indicadores da QFS, por estarem sujeitos às maiores alterações, em função dos sistemas de manejo.

Além dos indicadores de QS considerados quantitativos, há indicadores qualitativos que podem ser observados diretamente na propriedade. Sua análise deve ser realizada em momentos estratégicos durante o ciclo das culturas e alguns precisam ser observados ao longo do tempo (Reinert et al., 2006). Para Reichert et al. (2003), o indicador de qualidade de maior relevância é a observação de como as raízes "se relacionam" com o ambiente do solo abaixo da superfície, ou seja, a determinação do que se convencionou chamar de estudo do perfil das raízes.

Nos últimos anos muito empenho tem sido dedicado para geração de indicadores que possam avaliar a QFS. Conforme Karlen et al. (1997), um bom índice, para que possa ser utilizado na avaliação de um processo ou atributo, deve: (a) de algum modo influenciar o processo para o qual a avaliação está sendo feita; (b) permitir a comparação com alguma condição definida como padrão e; (c) ser sensível e permitir a detecção de diferenças em escala pontual no tempo e no espaço. Contudo, a maior dificuldade tem sido encontrar um índice que inclua os atributos físicos do solo e que se relacione com o crescimento, desenvolvimento e produção das plantas (Santos, 2010).

Sabendo que as propriedades físicas do solo são interdependentes e que, consequentemente, a modificação de uma delas normalmente leva à modificação de todas as demais, propõe-se uma divisão hierárquica dos indicadores de QFS a partir do grau relativo de dificuldade de obtenção de seus valores, sendo classificados (I) primários: aqueles mais facilmente mensuráveis, obtidos por medidas diretas de campo ou de laboratório e (II) secundários: aqueles que, normalmente, são derivados de dados obtidos no campo e/ou no laboratório (indicadores primários), mas que exigem certo grau de tratamento estatístico ou modelagem, sendo de mais difícil interpretação.
Todavia, quando se objetiva estudar os atributos que fazem parte da estabilidade estrutural, como indicadores da qualidade física do solo sob diferentes sistemas de manejo, a eficiência ou não da avaliação dependerá do monitoramento ao longo do tempo (Secco et al., 2005).

\section{Indicadores primários}

Na Tabela 1 são apresentados os principais indicadores primários da QFS. É essencial que os indicadores básicos de QS se relacionem com as funções do ecossistema, tais como ciclagem de carbono e nitrogênio, e devem ser propriedades mensuráveis, holísticas e acessíveis para usuários nos diferentes níveis.

Tabela 1. Principais atributos de solos utilizados como indicadores primários para monitorar a qualidade do solo e metodologias para a sua obtenção

\begin{tabular}{|c|c|}
\hline Atributo do solo & Metodologia \\
\hline Densidade do solo & Método do anel volumétrico \\
\hline $\begin{array}{l}\text { Resistência à } \\
\text { penetracão }\end{array}$ & Método do penetrômetro (índice de cone) \\
\hline Agregação (> 2mm) & Separação por peneiramento úmido \\
\hline Textura & $\begin{array}{l}\text { Método da pipeta ou densimétrico } \\
\text { Determinada a partir da umidade de saturação; }\end{array}$ \\
\hline Porosidade total & $\begin{array}{l}\text { e pela relação entre a densidade do solo e a } \\
\text { densidade de partículas }\end{array}$ \\
\hline Infiltração & Método do infiltrômetro de aneis \\
\hline $\begin{array}{l}\text { Condutividade } \\
\text { hidráulica saturada }\end{array}$ & $\begin{array}{l}\text { Método do pemeâmetro de carga constante } \\
\text { (laboratório) ou permeâmetro de Ghelph } \\
\text { (campo) }\end{array}$ \\
\hline
\end{tabular}

Fonte: Adaptado de Arshad \& Martin (2002)

\section{Indicadores secundários}

Curva de retenção de água (CRA): $A$ CRA tem-se revelado como atributo físico-hídrico importante nos estudos da QFS, com vistas a nortear as práticas de uso e o manejo sustentável dos sistemas agrícolas (Santos, 2010). As informações obtidas com a CRA possibilitam calcular valores de outros atributos do solo como, por exemplo, densidade do solo, porosidade total e sua distribuição, saturação efetiva, dentre outros, podendo demonstrar através de sua forma (complexidade/ sinuosidade) o estado estrutural do solo, refletindo claramente sua porosidade.

É relativamente simples de ser obtida do ponto de vista operacional e expressa importantes relações físico-hídricas da matriz do solo, sendo considerada como uma informação fundamental para o desenvolvimento e aplicação de indicadores da QFS (Silva et al., 1994), com conteúdo de água retido em determinado potencial decorrente da estrutura e da distribuição dos tamanhos de poros (Beutler et al., 2002).

Rawls et al. (1991) mostraram que, em elevados potenciais, a CRA é influenciada por poros estruturais associados ao efeito da matéria orgânica na formação e na estabilidade da estrutura do solo. Em baixo potencial, a composição granulométrica e a mineralogia do solo se tornam mais importantes devido à superfície disponível para a adsorção de água (Gupta \& Larson, 1979). Assim, Carducci et al. (2011) concluíram, estudando a modelagem da CRA de Latossolos no Cerrado, que o conteúdo de argila nesses solos influencia a retenção de água, elevando a capilaridade e adsorção de água. 
Machado et al. (2008) afirmam que apesar da maioria dos estudos descrever as relações entre as variáveis do solo e os coeficientes da equação da CRA numa ampla variação de solos, sob um mesmo solo os diferentes sistemas de uso e manejo também podem promover modificações na sua estrutura e no comportamento físico-hídrico do solo.

Marchão et al. (2007), avaliando diferentes sistemas agrícolas incluindo plantio direto e convencional, ajustaram o conteúdo relativo de água a um modelo não linear de curva de retenção de água (modelo de Assouline) e demonstraram que, apesar de em muitos casos a comparação utilizando a curva como um índice de qualidade física não levar a conclusões muito claras sobre o efeito do manejo, este atributo tem potencial devido a mudanças na forma da CRA relativa com a degradação do solo.

Os autores lembram que o fato de englobar diferentes atributos em um mesmo modelo complexo pode afetar a sensibilidade das curvas e inviabilizar sua utilização em comparações de sistemas de manejo; contudo, Santos (2010) e Santos et al. (2011a) observam que a interpretação correta dos diversos atributos físico-hídricos do solo, associada às suas inter-relações, permite avaliar com maior precisão a QFS nos mais variados sistemas de produção agropecuário, indicando o grau de degradação do solo em determinada área, quando comparada a solos sem alterações de ordem física.

Apesar de Chicota \& Lier (2004) apontarem que particularidades inerentes à variabilidade espacial das CRA e a variabilidade geral de seus pontos experimentais ainda não são bem descritas, uma CRA permite a interpretação das relações existentes entre a densidade do solo, teores de carbono orgânico, água do solo e da resistência à penetração em diferentes sistemas de uso e manejo de solo.

Parâmetro ou índice-S: Dexter \& Bird (2001) e Dexter (2004) propuseram um novo índice para se avaliar a QFS a partir da curva de retenção da água no solo, o "índice S". A teoria do índice-S como indicadora de qualidade física ou estrutural do solo, conforme apresentado por Dexter (2004) e Reynolds et al. (2009), teve como premissa essencial ser "determinada primariamente por manejos induzidos de poros na estrutura do solo (porosidade estrutural ou macroporos), ao invés de poros alterados na matriz do solo (porosidade textural ou microporos)".

Em grande parte, a estrutura dos poros determina a forma da curva de liberação de água do solo pela tensão presente entre a saturação $(\mathrm{h}=0)$ e o ponto de inflexão (hi); já os poros da matriz do solo, por outro lado, incluem os espaços dentro dos agregados (intra-agregados ou microporos) e entre partículas individuais de areia, silte, argila e matéria orgânica e estas, em grande parte, controlam a liberação de água do solo em maiores tensões do que a tensão do ponto de inflexão da CRA.

Desta forma, o índice-S está relacionado a variáveis como textura, densidade do solo, matéria orgânica e crescimento de raízes. Conceitualmente, o índice $\mathrm{S}$ equivale à inclinação da reta tangente ao ponto de inflexão da curva característica de retenção de água (Figura 1). É determinado pela CRA expressando-se o conteúdo gravimétrico de água $(\theta)$ em função da tensão de água no solo (h) após ajuste utilizando a equação de van Genuchten e o logaritmo natural da tensão da água no solo $\ln (\mathrm{h})$.

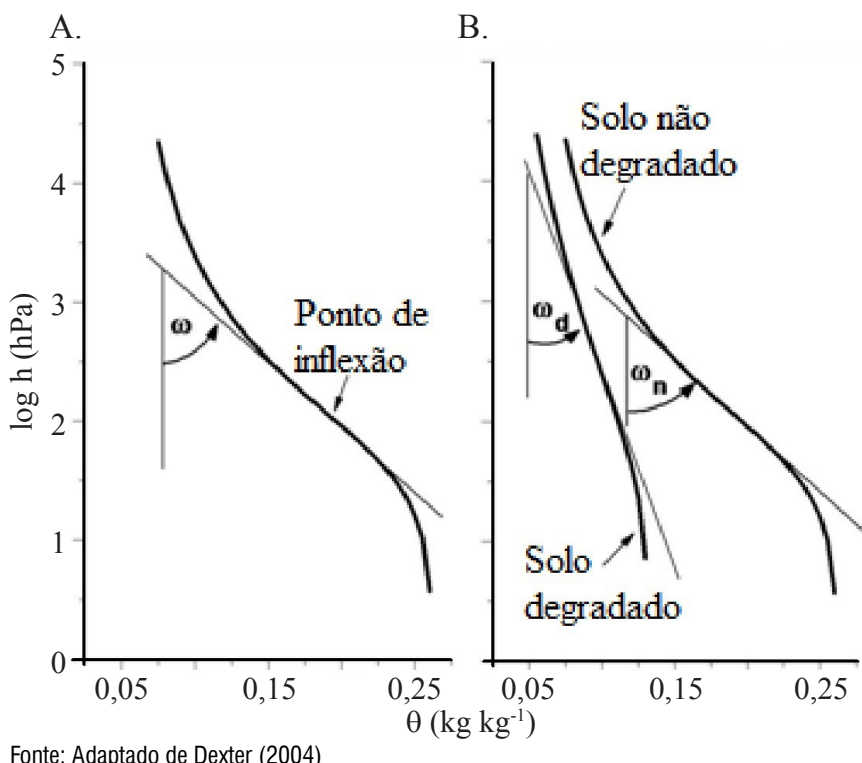

Figura 1. Representação esquemática das curvas características de retenção de água e seu ponto de inflexão (esquerda) e em duas condições de manejo com solo considerado degradado e não degradado (direita)

Assim, o uso e o manejo do solo podem ser avaliados e comparados por meio do índice-S na medida em que há alterações na distribuição do diâmetro dos poros (Pereira et al., 2011). Dexter (2004) sugeriu que valores do índice-S iguais a 0,035 deveriam ser utilizados como limite para se separar solos de boa qualidade estrutural dos solos de qualidade estrutural inferior, e que valores menores que 0,020 estariam associados com solos de péssima qualidade física.

Nesse sentido, Andrade \& Stone (2009) concluíram que o valor limite para o índice-S igual a 0,045 foi o mais adequado à divisão entre solo de boa qualidade estrutural e solo com tendências a se tornar degradado, enquanto valores do índice-S menores ou iguais a 0,025 indicam solos inteiramente degradados fisicamente. No entanto, de acordo com Reynolds et al. (2009), não está claro como este indicador de QFS pode ser beneficamente aplicado para solos argilosos e muito expansivos e pode, ainda, levar a indicações errôneas quando aplicado para solos de textura mais arenosa e outros materiais de estrutura granular simples, como é o caso da maioria dos Latossolos do Cerrado.

Inicialmente, Dexter \& Bird (2001) observaram que havia dois pontos de inflexão possíveis, dependendo se a CRA fosse construída plotando-se a umidade em função de $\ln (\mathrm{h})$ ou $\mathrm{h}$. Os autores indicaram que os dois pontos de inflexão estariam muito próximos para solos com estreita distribuição de poros por tamanho, independentemente da escala utilizada na função, para explicar o uso das curvas de $\theta$ traçadas apenas em função de $\ln (\mathrm{h})$ ou sua representação correspondente em termos de $\log (\mathrm{h})$.

Mais recentemente, Santos et al. (2011b) propuseram nova metodologia para obtenção do índice $\mathrm{S}$ mantendo-se a teoria inicialmente proposta por Dexter \& Bird (2001), porém, considerando para o cálculo a inclinação da CRA no seu ponto de inflexão em função da variável independente, quer seja $\mathrm{h}$, $\ln (\mathrm{h})$ ou $\log (\mathrm{h})$. Os autores demonstraram que a expressão da 
CRA em função de $\ln (\mathrm{h})$ ou $\log (\mathrm{h})$ ao invés de h como variável independente, resulta em valores diferentes do índice $\mathrm{S}$, o que pode limitar a discussão das propriedades físicas do solo.

Os novos modelos propostos por Santos et al. (2011b) para as diferentes escalas de ajuste são apresentados pelas Eqs. 1, 2 e 3 :

$$
\begin{gathered}
\mathrm{S}_{\mathrm{h}}=-(\hat{\alpha})(\hat{\mathrm{n}}-1)\left(\theta_{\mathrm{s}}-\hat{\theta}_{\mathrm{r}}\right)(\hat{\mathrm{m}})^{\hat{\mathrm{m}}}(1+\hat{\mathrm{m}})^{-\hat{\mathrm{m}}-1} \\
\mathrm{~S}_{\ln (\mathrm{h})}=-\left(\hat{\alpha}_{1}\right)\left(\hat{\mathrm{n}}_{1}-1\right)\left(\theta_{\mathrm{s}}-\hat{\theta}_{\mathrm{r} 1}\right)\left(\hat{\mathrm{m}}_{1}\right)^{\hat{\mathrm{m}}_{1}}\left(1+\hat{\mathrm{m}}_{1}\right)^{-\hat{\mathrm{m}}_{1}-1} \\
\mathrm{~S}_{\log (\mathrm{h})}=-\left(\hat{\alpha}_{2}\right)\left(\hat{\mathrm{n}}_{1}-1\right)\left(\theta_{\mathrm{s}}-\hat{\theta}_{\mathrm{r} 1}\right)\left(\hat{\mathrm{m}}_{1}\right)^{\hat{\mathrm{m}}_{1}}\left(1+\hat{\mathrm{m}}_{1}\right)^{-\hat{\mathrm{m}}_{1}-1}
\end{gathered}
$$

Intervalo hídrico ótimo (IHO): Letey (1985) propôs, na tentativa de obter um índice único que englobasse a QFS, o termo "Non-limiting Water Range" como uma faixa de teor de água que integra as propriedades físicas do solo diretamente relacionadas com o crescimento das plantas.

Posteriormente, este termo foi aprimorado por Silva et al. (1994) como "Least Limiting Water Range" (LLWR) e definido como a faixa de teor de água no solo em que as limitações relativas à disponibilidade de água, aeração e resistência à penetração, são mínimas, tornando-se um indicador da qualidade estrutural do solo para a produtividade das culturas. No Brasil, o "Least Limiting Water Range" tem sido comumente designado como Intervalo Hídrico Ótimo (Tormena et al., 1998).

O IHO foi definido como a faixa de conteúdo de água do solo determinada por dois limites: o superior, no qual a umidade do solo correspondente à capacidade de campo ou à umidade na qual a porosidade de aeração é de $10 \%$; e o limite inferior, cuja umidade corresponde ao ponto de murcha permanente ou à umidade na qual a resistência do solo à penetração atinge o valor de 2,0 MPa (Imhoff, 2002).

Esse indicador aprimora o conceito tradicional de água disponível entre capacidade de campo e o ponto de murcha permanente integrando o efeito do potencial matricial, aeração e resistência do solo à penetração das raízes em um único parâmetro, possibilitando estabelecer as condições de umidade do solo limitantes ao crescimento das plantas. Atualmente, tem sido utilizado para investigar os efeitos de sistemas de uso e manejo em modificações na QFS, sendo atestado como eficiente indicador para o monitoramento da compactação e QFS (Beutler et al., 2007), quando aplicado com base no valor de resistência à penetração limitante e no valor de densidade crítica do solo.

Diversos estudos já demonstraram que as práticas de manejo do solo afetam o IHO e que pode haver redução de crescimento das plantas com redução da sua amplitude. Por outro lado, os estudos relacionando o IHO com a produtividade das culturas são ainda incipientes (Sá et al., 2007).

Um fator que tem limitado a utilização do IHO e sua aplicação em estudos envolvendo QS é a necessidade de se obter valores de resistência à penetração em diferentes condições de umidade ou tensão de água no solo, sendo imprescindível a utilização de penetrômetros de bancada estáticos. Esses equipamentos são montados com sistemas elétrico-eletrônicos complexos que apresentam custo elevado, razão pela qual Sá et al. (2007) propuseram um minipenetrômetro de bancada que, além de apresentar eficácia comprovada, tem baixo custo.

Índice proposto por Reynolds et al. (2002): Reynolds et al. (2002) realizaram estudos similares ao de Skopp et al. (1990), usando indicadores e características em função do volume de poros para quantificar a QFS, e concluíram que os critérios propostos, $\mathrm{FC} / \mathrm{PORt}=0,66$ (FC: umidade volumétrica do solo; PORt: porosidade total do solo) e ACt/PORt $=0,34$ (ACt: aeração total do solo), para capacidade de armazenamento "ideal" de água e ar do solo foram úteis como bons indicadores de QFS. Foram coerentes com os critérios estabelecidos, como os usados para OC (carbono orgânico), BD (densidade do solo seco), AC (aeração do solo) e PAWC (umidade do ponto de murcha permanente), e incorporaram parâmetros físicos do solo adicionais (FC e PORt) e outros aspectos da QS (produção microbiana de nitrogênio), que ainda não haviam sido explícitos em critérios existentes.

Segundo esses autores, seriam necessários, ainda, mais estudos para se determinar a QS, caso a produtividade das culturas a campo e/ou de saúde ambiental rural mostrem evolução positiva e constante dos seus atributos, mantendo as relações apresentadas e os outros parâmetros do indicador dentro de seus respectivos intervalos ideais.

\section{Indicadores de Qualidade Física do Solo: Uma Análise CRítica}

Ao relacionar alguns índices de QFS existentes como, por exemplo, o índice proposto por Reynolds et al. (2002), verificam-se limitações ao uso deste indicador por ainda não haver número aceitável de resultados em diferentes localidades, suficientes para que se possa utilizá-lo como índice confiável na determinação da QFS. Ainda, esse índice não leva em consideração outros atributos físicos bastante sensíveis às diversas práticas de cultivo e manejo do solo sendo conveniente, também, a realização de um grande número de estudos em função do tipo de solo (Santos, 2010).

O índice de QFS, desenvolvido por Letey (1985) e caracterizado por Silva et al. (1994) como sendo o intervalo hídrico ótimo (IHO), tem apresentado bons resultados (Silva et al., 1994; Beutler et al., 2007; Lima et al., 2012), porém sua aplicação prática fica limitada a períodos em que o solo se encontra com níveis adequados de umidade para que a resistência do solo à penetração possa ser medida.

Ao se proceder a uma análise minuciosa do índice $S$ proposto por Dexter (2004), verifica-se que a expressão da CRA em função de $\ln (\mathrm{h})$ ou $\log (\mathrm{h})$, ao invés de h como variável independente, resulta em valores diferentes do índice S (Santos et al., 2011b). Dessa forma, o cálculo do índice $\mathrm{S}$ quando a CRAé expressa com $\mathrm{h}$ sendo a variável independente é mais consistente do ponto de vista matemático e físico e, para as amostras que esses autores selecionaram, esta forma de cálculo aumentou significativamente a relevância da análise em comparação ao $\mathrm{S}$ proposto por Dexter (2004). Por fim, de acordo com Santos et al. (2011b) e Pereira et al. (2011), mais estudos deverão ser conduzidos para verificar em que proporção o índice $\mathrm{S}$ é afetado em diferentes tipos de solos e sob diferentes manejos, visando verificar se o uso de h como 
variável independente efetivamente aumenta a sensibilidade da análise.

\section{Conclusões}

1. Ao discriminar solos com sinais de degradação, os indicadores de qualidade física do solo evidenciam a necessidade da adoção de sistemas que favoreçam a estruturação do solo, como aqueles que elevam os teores de matéria orgânica.

2. Os indicadores de qualidade física do solo existentes são gerados para situações específicas, demandando número maior de estudos para validação e aplicação confiável, o que configura oportunidade para o desenvolvimento de novos indicadores físicos de qualidade do solo.

3. Para uma gestão segura dos recursos naturais, a análise quantitativa e a interpretação dos atributos físicos e físicohídricos e das principais alterações geradas na qualidade do solo são fundamentais.

\section{Literatura Citada}

Aguiar, M. I. Qualidade física do solo em sistemas agroflorestais. Viçosa: UFV, 2008. 91p. Dissertação Mestrado

Albuquerque, J. A.; Sangoi, L.; Ender, M. Efeitos da integração lavoura-pecuária nas propriedades físicas do solo e características da cultura do milho. Revista Brasileira de Ciência do Solo, v.25, p.717-723, 2001.

Andrade, R. S.; Stone, L. F. Índice S como indicador da qualidade física de solos do cerrado brasileiro. Revista Brasileira de Engenharia Agrícola e Ambiental, v.13, p.382388, 2009.

Andreola, F.; Costa, L. M.; Olszevski, N. Influência da cobertura vegetal de inverno e da adubação orgânica e, ou, mineral sobre as propriedades físicas de uma Terra Roxa Estruturada. Revista Brasileira de Ciência do Solo, v.24, p.857-865, 2000.

Arnold, P. W. Surface-electrolyte interactions. In: Greenland, D. J.; Hayes, M. H. B. (ed.). The chemistry of soil constituents. Chichester: Wiley, 1978. p.355-404.

Arshad, M.A.; Martin, S. Identifying critical limits for soil quality indicators in agro-ecosystems. Agriculture, Ecosystems and Environment, v.88, p.153-160, 2002.

Bastos, R. S.; Sá Mendonça, E.; Alvarez V., V. H.; Corrêa, M. M.; Costa, L. M. Formação e estabilização de agregados do solo influenciados por ciclos de umedecimento e secagem após adição de compostos orgânicos com diferentes características hidrofóbicas. Revista Brasileira de Ciência do Solo, v.29, p.21-31, 2005.

Beutler, A. N.; Centurion, J. F.; Centurion, M. A. P. C.; Leonel, C. L.; João, A. C. G. S.; Freddi, O. S. Intervalo hídrico ótimo no monitoramento da compactação e da qualidade física de um Latossolo Vermelho cultivado com soja. Revista Brasileira de Ciência do Solo, v.31, p.1223-1232, 2007.

Beutler, A. N.; Centurion, J. F.; Roque, C. G.; Ferraz, M. V. Densidade relativa ótima de Latossolos Vermelhos para a produtividade de soja. Revista Brasileira de Ciência do Solo, v.29, p.843-849, 2005.
Beutler, A. N.; Centurion, J. F.; Souza, Z. M.; Andrioli, I.; Roque, C. G. Retenção de água em dois tipos de Latossolos sob diferentes usos. Revista Brasileira de Ciência do Solo, v.26, p.829-834, 2002.

Blainski, E.; Tormena, C. A.; Fidalski, J.; Guimarães, R. M. L. Quantificação da degradação física do solo por meio da curva de resistência do solo à penetração. Revista Brasileira de Ciência do Solo, v.32, p.975-983, 2008.

Braida, J. A.; Reichert, J. M.; Reinert, D. J.; Veiga, M. Teor de carbono orgânico e a susceptibilidade à compactação de um Nitossolo e Argissolo. Revista Brasileira de Engenharia Agrícola e Ambiental, v.14, p.131-139, 2010.

Camargo, O. A.; Alleoni, L. R. F. Compactação do solo e o desenvolvimento das plantas. Piracicaba: ESALQ/USP, 1997. 132p.

Campos, B. C.; Reinert, D. J.; Nicolodi, R.; Ruedell, J.; Petrere, C. Estabilidade estrutural de um Latossolo Vermelho-Escuro distrófico após sete anos de rotação de culturas e sistemas de manejo de solo. Revista Brasileira de Ciência do Solo, v.19, p.121-126, 1995.

Carducci, C. E.; Oliveira, G. C.; Severiano, E. C.; Zeviani, W. M. Modelagem da curva de retenção de água de Latossolos utilizando a Equação Duplo Van Genuchten. Revista Brasileira de Ciência do Solo, v.35, p.77-86, 2011.

Cassol, L. C. Relação solo-planta-animal num sistema de integração lavoura-pecuária em semeadura direta com calcário na superfície. Porto Alegre: UFRGS, 2003. 157p. Tese Doutorado

Chicota, R.; Lier, Q. J.; Análise da variabilidade espacial de pontos amostrais da curva de retenção da água no solo. Revista Brasileira de Ciência do Solo, v.28, p.585-596, 2004.

Collares, G. L.; Reinert, D. J.; Reichert, J. M.; Kaiser, D. R. Compactação de um Latossolo induzida pelo tráfego de máquinas e sua relação com o crescimento e produtividade do feijão e trigo. Revista Brasileira de Ciência do Solo, v.32, p.933-942, 2008.

Costa, A.; Albuquerque, J.; Mafra, A. L.; Silva, F. R. Propriedades físicas do solo em sistemas de manejo na integração lavoura-pecuária. Revista Brasileira de Ciência do Solo, v.33, p.235-244, 2009.

Dantas, J. d'A. N.; Oliveira, T. S.; Mendonça, E. S.; Assis, C. P. Qualidade do solo sob diferentes usos e manejos no perímetro irrigado de Jaguaribe/Apodi, CE. Revista Brasileira de Engenharia Agrícola e Ambiental, v.16, p.1826, 2012.

Dexter, A. R. Soil physical quality: Part I. Theory, effects of soil texture, density, and organic matter, and effects on root growth. Geoderma, v.120, p.201-214, 2004.

Dexter, A. R.; Bird, N. R. A. Methods for predicting the optimum and the range of water contents for tillage based on the water retention curve. Soil \& Tillage Research, v.57, p.203-212, 2001.

Doran, J. W.; Parkin, T. B. Defining and assessing soil quality. In: Doran, J. W.; Coeman, D. C.; Bezdicek, D. F.; Stewart, B. A. (ed.). Defining soil quality for sustainable environment. Madison: Soil Science Society of America, 1994. p.3-21. SSSA Special Publication, 35 
Dufranc, G.; Dechen, S. C. F.; Freitas, S. S.; Camargo, O. A. Atributos físicos, químicos e biológicos relacionados com a estabilidade de agregados em dois latossolos em plantio direto no Estado de São Paulo. Revista Brasileira de Ciência do Solo, v.28, p.505-517, 2004.

Ferreira, M. M. Caracterização física do solo. In: Lier, Q. J. van (ed.). Física do solo. Viçosa: Sociedade Brasileira de Ciência do Solo, 2010. p.1-27.

Figueiredo, C. C.; Santos, G. G.; Pereira, S.; Nascimento, J. L.; Alves Júnior, J. Propriedades físico-hídricas em Latossolo do Cerrado sob diferentes sistemas de manejo. Revista Brasileira de Engenharia Agrícola e Ambiental, v.13, p.146151,2009

Flowers, M. D.; Lal, R. Axle load and tillage effects on soil physical properties and soybean grain yield on a molic ochraqualf in northwest Ohio. Soil \& Tillage Research, v.48, p.21-35, 1998.

Guérif, J. Factors influencing compaction-induced increases in soil strength. Soil \& Tillage Research, v.16, p.167-178, 1990.

Gupta, S. C.; Larson, W. E. Estimating soil water retention characteristics from particle size distribution, organic matter percent, and bulk density. Water Research, v.15, p.1633-1635, 1979.

Hamza, M. A.; Anderson, W.K. Soil compaction in cropping systems: A rewiew of the nature, causes and possible solutions, Soil \& Tillage Research, v.82, p.121-145, 2005.

Helalia, A. M.; Letey, J.; Graham, R. C. Crust formation and clay migration effects on infiltration rate. Soil Science Society of American Journal, v.52, p.251-255, 1988.

Hillel, D. Fundamentals of soil physics. New York: Academic Press, 1980. 413p.

Imhoff, S. D. C. Indicadores de qualidade estrutural e trafegabilidade de Latossolos e Argissolos Vermelhos. Piracicaba: ESALQ/USP, 2002. 94p. Tese Doutorado

Karlen, D. L.; Mausbach, M. J.; Doran, J. W.; Cline, R. G.; Harris, R. F.; Chuman, G. E. Soil quality: A concept, definition, and framework for evaluation. Soil Science Society of American Journal, v.61, p.4-10, 1997.

Kemper, W. D.; Rosenau, R. C.; Dexter, A. R. Cohesion development in disrupted soils as affected by clay and organic matter content and temperature. Soil Science Society of America Journal, v.51, p.860-867, 1987.

Kiehl, E. J. Manual de edafologia. São Paulo: Agronômica Ceres, 1979. 262p.

Lanzanova, M. E.; Nicoloso, R. S.; Lovato, T.; Eltz, F. L. F.; Amado, T. J. C.; Reinert, D. J. Atributos físicos do solo em sistema de integração lavoura-pecuária sob plantio direto. Revista Brasileira de Ciência do Solo, v.31, p.1131-1140, 2007.

Leão, T. P. Intervalo hídrico ótimo em diferentes sistemas de pastejo e manejo da pastagem. Piracicaba: ESALQ/USP, 2002. 58p. Dissertação Mestrado

Letey, J. Relationship between soil physical properties and crop production. Advances in Soil Science, v.1, p.277-294, 1985.

Libardi, P. L. Dinâmica da água no solo. 2.ed. Piracicaba: ESALQ/USP, 2000. 509p.
Lima, V. M. P.; Oliveira, G. C.; Serafim, M. E.; Curi, N.; Evangelista, A. R. Intervalo hídrico ótimo como indicador da melhoria da qualidade estrutural de Latossolo degradado. Revista Brasileira de Ciência do Solo, v.36, p.71-78, 2012.

Macedo, M. C. M. Integração lavoura pecuária: O estado da arte e inovações tecnológicas. Revista Brasileira de Zootecnia, v.38, p.133-146, 2009.

Machado, J. L.; Tormena, C. A.; Fidalski, J.; Scapim, C. A. Inter-relações entre as propriedades físicas e os coeficientes da curva de retenção de água de um Latossolo sob diferentes sistemas de uso. Revista Brasileira de Ciência do Solo, v.32, p.495-502, 2008.

Marchão, R. L.; Balbino, L. C.; Silva, E. M.; Santos Júnior, J. D. G.; Sá, M. A. C.; Vilela, L.; Becquer, T. Qualidade física de um Latossolo Vermelho sob sistemas de integração lavourapecuária no cerrado. Pesquisa Agropecuária Brasileira, v.42, p.873-882, 2007.

MEA - Millennium Ecosystem Assessment. Ecosystem and human well-being: Synthesis. Washington: Island Press, 2005. 137p.

Moreira, W. H.; Betioli Júnior, E.; Petean, L. P.; Tormena, A. C.; Alves, S. J.; Costa, M. A. T.; Franco, H. H. S. Atributos físicos de um Latossolo Vermelho distroférrico em sistema de integração lavoura-pecuária. Revista Brasileira de Ciência do Solo, v.36, p.389-400, 2012.

Muggler, C. C.; Pape, T. H.; Buurman, P. Laser grain-size determination in soil genetic studies 2. Clay content, clay formation, and aggregation in some Brazilian Oxisols. Soil Science, v.162, p.219-228, 1997.

Pereira, F. S.; Andrioli, I.; Pereira, F. S.; Oliveira, P. R.; Centurion, J. F.; Falqueto, R. J.; Martins, A. L. S. Qualidade física de um Latossolo Vermelho submetido a sistemas de manejo avaliado pelo índice S. Revista Brasileira de Ciência do Solo, v.35, p.87-95, 2011.

Rawls, W. J.; Gish, T. J.; Brakensiek, D. L. Estimating soil water retention from soil physical properties and characteristics. Advances in Soil Science, v.16, p.213-234, 1991.

Reichert, J. M.; Reinert, J. M.; Braida, J. A. Qualidade dos solos e sustentabilidade de sistemas agrícolas. Ciência \& Ambiente, v.27, p.29-48, 2003.

Reinert, D. J.; Reichert, D. J.; Veiga, M.; Suzuki, L. E. A. S. Qualidade física dos solos. In: Reunião Brasileira de Manejo e Conservação do Solo e da Água, 16, 2006. Aracaju, Resumos... Aracaju: SBCS, 2006. CD-Rom

Reynolds, W. D.; Bowman, B. T.; Drury, C. F.; Tan, C. S.; Lu, X. Indicators of good soil physical quality: density and storage parameters. Geoderma, v.110, p.131-146, 2002.

Reynolds, W. D.; Drury, C. F.; Tan, C. S.; Fox, C. A.; Yang, $\mathrm{X}$. M. Use of indicators and pore volume-function characteristics to quantify soil physical quality. Geoderma, v.152, p.252-263, 2009.

Ribon, A. A.; Tavares Filho, J. Estimativa da resistência mecânica à penetração de um Latossolo Vermelho sob cultura perene no norte do Estado do Paraná. Revista Brasileira de Ciência do Solo, v.32, p.1817-1825, 2008.

Rosolem, C. A.; Fernandez, E. M.; Andreotti, M.; Crusciol, C. A. C. Crescimento radicular de plântulas de milho afetado pela resistência do solo à penetração. Pesquisa Agropecuária Brasileira, v.34, p.821-828, 1999. 
Sá, M. A. C.; Santos Junior, J. D. G.; Resck, D. V. S. Ferreira, E. A. B.; Franz, C. A. B. Minipenetrômetro dinâmico para determinação da resistência à penetração em amostras de solo indeformadas. Pesquisa Agropecuária Brasileira, v.42, p.1659-1662, 2007.

Santos, G. G. Impacto de sistemas de integração lavourapecuária na qualidade física do solo. Goiânia: UFG, 2010. 122p. Tese Doutorado

Santos, G. G.; Marchão, R. L.; Silva, E. M.; Silveira, P. M.; Becquer, T. Qualidade física do solo sob sistemas de integração lavoura-pecuária. Pesquisa Agropecuária Brasileira, v.46, p.1339-1348, 2011 a.

Santos, G. G.; Silva, E. M.; Marchão, R. L.; Silveira, P. M.; Bruand, A.; James, F.; Becquer, T. Analysis of physical quality of soil using the water retention curve: Validity of the S-index. C. R. Geoscience, v.343, p.295-301, $2011 \mathrm{~b}$.

Secco, D.; Ros, C. O. da; Secco, J. K.; Fiorin, J. E. Atributos físicos e produtividade de culturas em um Latossolo Vermelho argiloso sob diferentes sistemas de manejo. Revista Brasileira de Ciência do Solo, v.29, p.407-414, 2005.

Silva, A. J. N.; Cabeda, M. S. V.; Lima, F. W. F. Efeito de sistemas de uso e manejo nas propriedades físico-hídricas de um Argissolo Amarelo de tabuleiro costeiro. Revista Brasileira de Ciência do Solo, v.29, p.833-842, 2005.
Silva, A. P.; Kay, B. D.; Perfect, E. Characterization of de least limiting water range. Soil Science Society of America Journal, v.58, p.1775-1781, 1994.

Singer, M.; Ewing, S. Soil quality. In: Sumner, M. E. (ed.). Handbook of soil science. Boca Raton: CRC Press, 2000. p.271-298.

Skopp, J.; Jawson, M. D.; Doran, J. W. Steady-state aerobic microbial activity as a function of soil water content. Soil Science Society of America Journal, v.54, p.1619-1625, 1990.

Tisdall, J. M.; Oades, J. M. The effect of crop rotation on aggregation in a red-brown earth. Australian Journal of Soil Research, v.18, p.423-433, 1980.

Tisdall, J. M.; Oades, J. M. Organic matter and water-stable aggregates in soils. Journal of Soil Science, v.33, p.141163, 1982.

Tormena, C. A.; Fidalski, J.; Rossi Junior, W. Resistência tênsil e friabilidade de um Latossolo sob diferentes sistemas de uso. Revista Brasileira de Ciência do Solo, v.32, p.33-42, 2008.

Tormena, C. A.; Silva, A. P.; Libardi, P. L. Caracterização do intervalo hídrico ótimo de um Latossolo Roxo sob plantio direto. Revista Brasileira de Ciência do Solo, v.22, p.573581, 1998.

Vezzani, F. M.; Mielniczuk, J. Agregação e estoque de carbono em Argissolo submetido a diferentes práticas de manejo agrícola. Revista Brasileira de Ciência do Solo, v.35, p.213223, 2011. 